\title{
The Prediabetes Outcome at National Guard Primary Health Care Centers in Riyadh, Saudi Arabia: Retrospective Chart Review
}

Mohammed A. Alateeq ${ }^{1}$, Moath Aljohani ${ }^{2}$, Sondos S. Kinani ${ }^{3}$, Ibrahim A. Aljabr ${ }^{4}$, Abdullah A. Alduayji ${ }^{4}$, Abdulrhman Aloud ${ }^{4}$, Elham Alzahrani ${ }^{3}$, Khalid Alharbi ${ }^{4}$

1. Family Medicine, Ministry of National Guard-Health Affairs, King Saud Bin Abdulaziz University for Health Sciences, Riyadh, SAU 2. Family and Community Medicine, College of Medicine and Medical Sciences, Qassim University, Qassim, SAU 3. Family Medicine, College of Medicine, King Saud Bin Abdulaziz University for Health Sciences, Riyadh, SAU 4. Family Medicine, College of Medicine and Medical Sciences, Qassim University, Qassim, SAU

Corresponding author: Moath Aljohani, m.aljohani@qu.edu.sa

\section{Abstract}

\section{Objectives}

To identify the outcome of prediabetes and the interventions that have been implemented for prediabetic patients at primary healthcare centers (PHCs) affiliated with King Abdulaziz Medical City, Riyadh, Saudi Arabia.

\section{Methodology}

This retrospective chart-review study was carried out using the BestCare electronic health records (EHRs) system. Data from the PHCs of King Abdulaziz Medical City, Riyadh, Saudi Arabia were extracted. Inclusion criteria were patients with prediabetes who were diagnosed between January 2015 and December 2016, with at least one follow-up visit. Variables included demographics, comorbidities, blood sugar lab results, and lipid profile measurements at each visit and intervention at the time of the initial diagnosis. Fisher's Exact test, sign test, and Kruskal-Wallis test were used to assess the differences for non-normally-distributed variables, while a paired t-test was conducted for paired and normally distributed continuous variables. Data were analyzed using the statistical program SAS, version 9.4 (SAS Institute Inc. Cary, NC).

\section{Result}

Of the 92 patients followed up with for three years, $76.08 \%$ remained in the prediabetic range, while $16.4 \%$ regressed to a normal glycemic state (NGS) and 7.6\% progressed to the diabetic range after intervention and follow-up for three years. Metformin use was not significant in the glycemic outcome. In comparison to the baseline, there was a considerable reduction in fasting blood sugar (FBS) and glycosylated hemoglobulin A1c (HbA1c) at the end of the follow-up.

Received 07/20/2020

Review began $07 / 21 / 2020$ Review ended 08/31/2020 Published 09/03/2020

\section{(c) Copyright 2020}

Alateeq et al. This is an open access article distributed under the terms of the Creative Commons Attribution License CC-BY 4.0., which permits unrestricted use, distribution, and reproduction in any medium, provided the original author and source are credited.

\section{Conclusion}

We found that most of the patients remained in the prediabetic range after the three-year follow-up, with or without intervention. A commonly prescribed pharmacological intervention like metformin showed no regression benefit in most patients. More extensive prospective studies are needed to evaluate the outcome and adherence to different interventions.

Categories: Endocrinology/Diabetes/Metabolism, Family/General Practice, Preventive Medicine Keywords: outcome, primary care, metformin, prediabetes

\section{Introduction}

Prediabetes is a serious health condition in which the glucose level is elevated but not enough for a diagnosis of diabetes [1]. The American Diabetic Association (ADA) defines prediabetes as fasting blood sugar (FBS) of 100-125 mg/dL (5.6-6.9 mmol/L), impaired glucose tolerance (IGT) 2-hour plasma glucose (2h PG) during 75-g oral glucose tolerance test (OGTT) of $140 \mathrm{mg} / \mathrm{dL}$ (7.8 mmol/L) to $199 \mathrm{mg} / \mathrm{dL}(11.0 \mathrm{mmol} / \mathrm{L})$, or glycosylated hemoglobulin A1c (HbA1c) of 5.7\%-6.4\% (39-47 mmol/mol) [2].

In Saudi Arabia, $25.5 \%$ of the population aged $\geqslant 30$ years is living with prediabetes, placing more than three million people at risk for diabetes mellitus (DM) [3,4]. An alarming figure is that $5 \%-10 \%$ of prediabetic people annually progress to type 2 diabetes mellitus (T2DM); in addition, $40.3 \%$ of diabetic patients are unaware that they have the disease $[3,5]$. Studies showed an increased risk of cardiovascular disease and allcause mortality among prediabetics [6]. Additionally, progression to DM poses a significant threat to the health care budget in Saudi Arabia, as DM accounts for 13.9\% of total health expenditure [4]. Therefore, the study of prediabetes outcomes aligns with the Saudi national transformation plan's strategic goals to 
increase efficiency and promote the prevention of health risks [7]. For that, a multi-dimensional control program/intervention approach is urgently needed to reverse the burden of diabetes in Saudi Arabia [4].

While different treatment modalities for DM exist, prevention is still the cornerstone of slowing down the escalating burden of diabetes [2]. Fortunately, $80 \%$ of cases of T2DM are preventable by simple interventions [4]. Recommended interventions include lifestyle interventions, which include $150 \mathrm{~min} /$ week of physical activity and a dietary recommendation to achieve $7 \%$ weight loss and improve insulin sensitivity [8]. A diabetes prevention program (DPP) trial provided the most robust evidence that a significant lifestyle modification could decrease the incidence of T2DM by $58 \%$ over the course of three years [9].On the other hand, pharmacological interventions are recommended for particularly high-risk groups; the most common intervention is metformin, which showed similar effectiveness in an Indian diabetic prevention study [10].

Identifying the outcome and intervention undertaken for prediabetics is useful for planning and implementing effective diabetes prevention programs and decreasing the burden of diabetes around the country [11,12]. Many studies have assessed the status of prediabetes in the Saudi population. Most of these studies focused on the prevalence and risk factors of diabetes $[12,13]$. There is a paucity of studies that focus on prediabetes outcomes and effective intervention after a follow-up period using retrospective or prospective data. Therefore, we conducted this multicenter retrospective chart review in primary health care centers (PHCs) affiliated with King Abdulaziz Medical City, National Guard, Riyadh to identify the outcome of prediabetes in the actual setting. Furthermore, we determined which intervention was taken to slow the progression to DM.

\section{Materials And Methods Study design and setting}

This retrospective chart review was conducted to identify the outcome of prediabetes and the documented interventions for prediabetic patients in the National Guard PHCs from the beginning of January 2015 to the end of December 2016, with a follow-up period of three years. The centers included the ambulatory care center (ACC), King Abdulaziz City Housing (Iskan Al Yarmouk), the National Guard Comprehensive Specialized Clinic (NGCSC), the Health Care Specialty Center (HCSC), the Employee Health Clinic (EHC), King Saud City Housing (Dirab clinics), and the King Abdullah Specialist Children's Hospital primary care clinic. This study was reviewed and approved by the Institutional Review Board (IRB) of the King Abdullah International Medical Research Center (KAIMRC). The confidentiality of data was guaranteed, and data were not disclosed unless for study purposes.

\section{Participants}

The inclusion criteria included all EHRs at National Guard PHCs in Riyadh for adults who were diagnosed with prediabetes as recommended by the ADA guidelines, i.e., FBS from $5.6 \mathrm{mmol} / \mathrm{L}$ to $6.9 \mathrm{mmol} / \mathrm{L}$ and HbA1c between 5.7\%-6.4\% [2], diagnosed in the period between January 1, 2015 and December 31, 2016 and has had at least one follow-up visit after the initial visit. The exclusion criteria were a diagnosis of DM or a normal glycemic state.

\section{Data collection method}

Two trained data collectors extracted data from EHRs in the Best Care system at National Guard PHCs. The baseline characteristics of the data included: sociodemographic data (age, sex), body mass index (BMI), smoking status, medication history at baseline, hypertension (by diagnosis or current use of antihypertensive medication), history of gestational diabetes, polycystic ovary syndrome, dyslipidemia (by diagnosis or on lipid-lowering agents), and cardiovascular disease (CVD). Blood sample results at the time of diagnosis and on each visit included the lipid profile in serum (total cholesterol [TC], low-density lipoprotein cholesterol [LDL], high-density lipoprotein cholesterol [HDL], and triglyceride [TG]) and blood sugar results (FBS, OGTT, and HbA1c). FBS, HbA1c, and the lipid profile were measured by auto-analyzers at the in-house laboratory of each local primary health care center. Data related to interventions like metformin, referral to bariatric surgery, and referral to the dietician at baseline were also included. Because of the nature of the study design, lifestyle changes implemented by patients such as exercise level or dietary changes were not tracked.

\section{Follow-up}

The study included only those individuals who were available for follow-up testing. Patients were followed up with from the time of initial diagnosis to the last available visit with available testing. Patients had variable frequency of follow-up visits depending on their medical status and presence of other comorbidities as assessed by the treating physician. If the patient had two initial abnormal tests, i.e., FBS and HBa1c, the patient was followed up based on their last recorded test. If only a single test had one abnormal result, i.e., FBS or HbA1c alone, the patient was followed up based on that abnormal tests.

\section{Outcome assessment}




\section{Cureus}

Outcome assessment was judged based on the patient's last available blood glucose measurement according to ADA guidelines [2]. A patient was considered prediabetic if their FBS was $100 \mathrm{mg} / \mathrm{dL}(5.6 \mathrm{mmol} / \mathrm{L})$ to 125 $\mathrm{mg} / \mathrm{dL}$ (6.9 mmol/L), if their IGT2-h PG during 75-g OGTT was $140 \mathrm{mg} / \mathrm{dL}$ (7.8 mmol/L) to $199 \mathrm{mg} / \mathrm{dL}(11.0$ $\mathrm{mmol} / \mathrm{L})$ or if their glycosylated HbA1c was $5.7 \%-6.4 \%$ (39-47 mmol/mol). If the patient's last readings were above the aforementioned, the patient was considered to be in the diabetic range. If the patient's readings were less, the patient was considered to be in the normal glycemic state (NGS).

\section{Statistical analysis}

Data were analyzed using the statistical program SAS (version 9.4, SAS Institute Inc., Cary, NC). Descriptive statistics were used to describe the study population at the baseline. Continuous variables were presented as mean and standard deviation (SD) or as median (IQR). Frequencies and proportions were calculated for categorical variables (gender, smoking, comorbidities, and type of intervention). Fisher's Exact test was used to determine associations between categorical variables, while the Kruskal-Wallis test was used for nonnormally distributed continuous variables. A sign test was used to compare non-normally distributed continuous variables and the paired t-test with normally distributed continuous variables. Statistical tests were considered significant at $\mathrm{p}<0.05$.

\section{Results}

In total, 102 participants were extracted from BestCare EHR. Ten of them were excluded: five had diabetes at the baseline, three were followed up with at the hospital rather than at PHCs, and two had no follow-up visits. The other 92 were followed up with as early as January 2015 to December 2019 until the last recorded visit. Table 1 shows that the mean age of the participants at the time of recruitment was $48.32 \pm 11.23$ years. Females constituted more than half of the sample (54.35\%). Overall, participants were either overweight or obese (31.9 \pm 5.89 ). Co-morbidities such as hypertension (61.96\%) and dyslipidemia (82.61\%) were prevalent among study individuals. Initial FBS was $6 \pm 0.55$, while HbA1c was $6.08 \pm 0.47$. The most common intervention undertaken was metformin, though one patient was already using metformin at baseline. None of the participants were prescribed another antidiabetic medication such as glucagon-like-peptide-1 receptor agonist (GLP-1) and sulfonylurea. Other demographics and clinical characteristics of participants are shown in Table 1.

\begin{tabular}{|c|c|c|}
\hline Variable & $\mathbf{N}$ & (\%) \\
\hline Age (mean \pm standard deviation (SD)) & \multicolumn{2}{|c|}{$48.3 \pm 11.2$} \\
\hline \multicolumn{3}{|l|}{ Gender } \\
\hline Male & 42 & 45.6 \\
\hline Female & 50 & 54.3 \\
\hline BMI initial (mean \pm standard deviation (SD)) & \multicolumn{2}{|c|}{$31.9 \pm 5.89$} \\
\hline \multicolumn{3}{|l|}{ Smoking } \\
\hline No & 84 & 91.3 \\
\hline Yes & 8 & 8.7 \\
\hline \multicolumn{3}{|l|}{ Hypertension } \\
\hline No & 35 & 38.0 \\
\hline Yes & 57 & 61.9 \\
\hline \multicolumn{3}{|l|}{ History of GDM,(women, $n=50)$. } \\
\hline No & 50 & 100 \\
\hline Yes & 0 & 0 \\
\hline \multicolumn{3}{|l|}{ PCOS (women, $n=50)$. } \\
\hline No & 46 & 95.6 \\
\hline Yes & 4 & 4.3 \\
\hline \multicolumn{3}{|l|}{ Dyslipidemia } \\
\hline No & 16 & 17.3 \\
\hline Yes & 76 & 82.6 \\
\hline
\end{tabular}




\section{Cureus}

CVD

No

Lab readings on Initial visit:

FBS (mmol/L)

HbA1c ( \%)

LDL (mmol/L)

Triglyceride (mmol/L)

$\mathrm{HDL}(\mathrm{mmol} / \mathrm{L})$

Total-cholesterol (mmol/L)

Intervention:

Metformin

No

Referral to the bariatric surgery

No

Yes

Referral to dietician

No

TABLE 1: Demographics and baseline clinical characteristics $(n=92)$

Values are presented as mean \pm standard deviation (SD), or numbers and percentages (\%).

BMI: body mass index. GDM: gestational diabetes mellitus, PCOS: polycystic ovarian syndrome, CVD: cardiovascular disease, FBS: fasting blood sugar, HbA1c:nhemoglobin A1c, LDL: low-density lipoprotein cholesterol, HDL: high-density lipoprotein cholesterol.

Figure 1 is a bar chart showing 92 prediabetic participants at the first visit. It presents the transformation to different glycemic states along with follow-up, with the median number of six visits for study participants and a median follow-up period of 44 months. Patients had various frequency of visits depending on many factors including the glycemic status and presence of other comorbidities as determined by the treating physician.

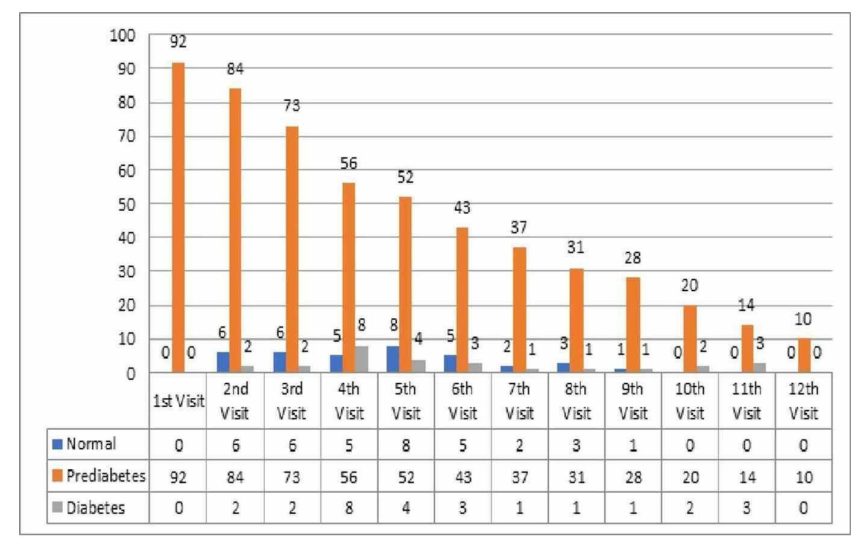

FIGURE 1: Frequencies and proportions presented indicate the number of follow-ups at each visit among prediabetics in different glycemic 


\section{Cureus}

\section{states}

The $x$-axis represents the number of visits and the $y$-axis represents the proportion of study participants at each follow-up visit.

Table 2 shows the accomplishment of the objective of this study, which was to identify the prediabetes outcome after various interventions. The majority of the participants (76.08\%) remained prediabetic, $16.30 \%$ regressed to NGS, and 7.6\% progressed to diabetes. Age, gender, and initial BMI showed no significant differences in various glycemic groups, although it is worth noting that the age of individuals in diabetes group $(41.71 \pm 9.3$, mean \pm SD) was less than that of NGS $(48.2 \pm 12.89)$ and female with NGS twice higher than male. Furthermore, co-morbidities such as hypertension and dyslipidemia were not shown to be associated with either outcome. However, we found that $66.67 \%$ of patients who regressed to NGS had hypertension, which equals two-thirds of NGS patients. Also, we found that $86.67 \%$ of patients who regressed to NGS had dyslipidemia.

Regarding smoking, we found that two (13.33\%) smokers, in contrast to 13 non-smokers (86.67\%), regressed to NGS, while seven (100\%) progressed to DM. Interestingly, about $64.13 \%$ took metformin; however, we found that only $16.9 \%$ of them regressed to NGS, while $76.3 \%$ remained at the prediabetic range and $6.8 \%$ progressed to diabetes. This showed that metformin produced no significant difference among the outcome groups. As expected, we found a significant difference between different glycemic groups in FBS and HbA1c at the end of follow-up, $\mathrm{p}<0.001$.

\begin{tabular}{|c|c|c|c|c|}
\hline Variable & Normoglycemia $(\mathrm{N}=15)$ & Prediabetes $(\mathrm{N}=70)$ & Diabetes $(\mathbf{N}=7)$ & P-Value \\
\hline Age & $48.2 \pm 12.89$ & $49 \pm 10.97$ & $41.71 \pm 9.3$ & 0.203 \\
\hline Gender & & & & 0.329 \\
\hline Male & 5 (33.33) & $35(50.00)$ & $2(28.57)$ & \\
\hline Female & $10(66.67)$ & $35(50.00)$ & $5(71.43)$ & \\
\hline BMI initial & $33.38 \pm 7.67$ & $31.21 \pm 5.36$ & $35.73 \pm 5.89$ & 0.113 \\
\hline Smoking & & & & 0.806 \\
\hline No & $13(86.67)$ & $64(91.43)$ & $7(100.00)$ & \\
\hline Yes & 2 (13.33) & $6(8.57)$ & $0(0.00)$ & \\
\hline Hypertension & & & & 0.934 \\
\hline No & $5(33.33)$ & $27(38.57)$ & $3(42.86)$ & \\
\hline Yes & $10(66.67)$ & $43(61.43)$ & $4(57.14)$ & \\
\hline PCOS (women) & & & & 0.240 \\
\hline No & 14 (93.33) & $68(97.14)$ & $6(85.71)$ & \\
\hline Yes & $1(6.67)$ & $2(2.86)$ & $1(14.29)$ & \\
\hline Dyslipidemia & & & & 0.188 \\
\hline No & $2(13.33)$ & $11(15.71)$ & $3(42.86)$ & \\
\hline Yes & $13(86.67)$ & 59 (84.29) & 4 (57.14) & \\
\hline CVD & & & & 1.000 \\
\hline No & 14 (93.33) & $63(90.00)$ & $7(100.00)$ & \\
\hline Yes & $1(6.67)$ & $7(10.00)$ & $0(0.00)$ & \\
\hline \multicolumn{5}{|l|}{ Final visit } \\
\hline FBS (mmol/L) & $5.28 \pm 0.35$ & $5.94 \pm 0.59$ & $7.76 \pm 0.81$ & $<0.001^{*}$ \\
\hline HbA1c (\%) & $5.51 \pm 0.07$ & $5.98 \pm 0.48$ & $7.22 \pm 0.37$ & $<0.001^{*}$ \\
\hline LDL (mmol/L) & $2.6 \pm 0.66$ & $2.76 \pm 0.96$ & $2.78 \pm 0.59$ & 0.926 \\
\hline Triglyceride (mmol/L) & $1.34 \pm 0.73$ & $1.29 \pm 0.54$ & $1.55 \pm 1$ & 0.937 \\
\hline
\end{tabular}




\section{Cureus}

HDL (mmol/L)

Total-cholesterol (mmol/L)

$1.11 \pm 0.25$

$4.3 \pm 0.85$

Intervention:

Metformin

No

Yes

$10(66.67)$

Referral for the bariatric surgery

No

Yes

Referral to dietician

No

Yes

14 (93.33)
$1.21 \pm 0.52$

$4.4 \pm 0.86$

$0.97 \pm 0.19$

0.142

$4.25 \pm 0.52$

0.813

0.931

3 (42.86)

4 (57.14)

0.671

67 (95.71)

7 (100.00)

$0(0.00)$

0.392

59 (84.29)

5 (71.43)

11 (15.71)

2 (28.57)

TABLE 2: Comparison of various sociodemographic, clinical characteristics and comorbidity variables based on response across "glycemic range at last visit"

Values are presented as mean \pm standard deviation (SD) or numbers and percentages (\%). Tests used: Fisher's exact test was used to determine associations between categorical variables, while the Kruskal-Wallis test was used for non-normally distributed continuous variables.

BMI: body mass index, GDM: gestational diabetes mellitus, PCOS: polycystic ovarian syndrome, CVD: cardiovascular disease, FBS: fasting blood sugar, HbA1c: hemoglobin A1c, LDL: low-density lipoprotein cholesterol, HDL: high-density lipoprotein cholesterol. *Statistically significant at $p<0.05$.

Table 3 shows the lipid profiles, glycemic indices, and BMI at the final visit. Sign and paired t-tests were used to assess the difference between the pre- and post-intervention groups. BMI showed an average increase of $0.4 \mathrm{~kg} / \mathrm{m} 2$ from the baseline, while HbA1c showed a significant decrease, with $\mathrm{p}<0.001$. Other parameters, including FBS, showed some improvement, though this was not significant, $\mathrm{p}>0.05$.

\begin{tabular}{|c|c|c|c|}
\hline Variable & Initial visit & Last visit & p-value \\
\hline BMI $\left(\mathrm{kg} / \mathrm{m}^{2}\right)$ & $31.0(7.89)$ & $31.4(7.28)$ & 0.525 \\
\hline FBS (mmol/L) & $5.9(0.65)$ & $5.8(0.8)$ & 0.050 \\
\hline HbA1c & $6.1(0.3)$ & $5.9(0.5)$ & $0.001^{*}$ \\
\hline LDL (mmol/L) & $2.7(1.23)$ & 2.6(1.07) & 0.428 \\
\hline Triglyceride (mmol/L) & $1.2(0.77)$ & $1.2(0.55)$ & 0.114 \\
\hline HDL (mmol/L) & $1.1 \pm 0.46$ & $1.1 \pm 0.48$ & 0.396 \\
\hline Total-cholesterol (mmol/L) & 4.4(1.39) & 4.2(1.06) & 0.301 \\
\hline
\end{tabular}

TABLE 3: Overall comparison between pre- and post-continuous variable at baseline and the end of the follow-up

Values are presented as mean \pm standard deviation (SD) for HDL and median (IQR) for non-normally distributed data. Test used: Sign test for association between continuous variables with non-normally distributed and paired t-test for associations between continuous variables with normally distributed variables.

BMI: body mass index, FBS: fasting blood sugar, HbA1c: hemoglobin A1c, LDL: low-density lipoprotein cholesterol, HDL: high-density lipoprotein cholesterol. * Statistically significant at $p<0.05$.

\section{Discussion}

This study aimed to identify the prediabetes outcome in PHCs setting in Saudi Arabia. The study found that most of the patients, $84 \%$, remained prediabetic or progressed to DM after three years of follow-up. This 
indicates a significant issue with prediabetics and their outcomes. This aligns with findings of Whitehall II cohort study in prediabetics defined by HbA1c, but not with FBS defined prediabetes, where it showed better reversion to normoglycemia [14]. However, our analysis lacks the comparison between prediabetics diagnosed based on FBS or HbA1c which in turn limits our ability to discuss which one of the tests had more reversion rates among prediabetics in the current study. In both studies, none of the patients were followed by OGTT, beyond pregnancy; OGTT has largely been eliminated from use in the diagnosis of diabetes, particularly due to the recent widespread standardization of the HbA1c assay and considering that our sample lacked any females with a GDM history [14]. Further studies among south Asian populations in 2015 demonstrated higher rates, with $58.9 \%$ of prediabetics progressing to DM; those higher rates can be attributed to the 10-year follow-up, as it is known that the yearly incidence of DM reaches up to $11 \%[15,16]$. In the neighboring Gulf states, it reported that UAE which has a prevalence of DM comparable to that of Saudi Arabia, has an incident rate of DM 4.8 incident cases/1,000 person-years, other studies reported higher rates as 15.2 per 1000 person-years in overweight and obese individuals; all in retrospective studies $[17,18]$.

This study shows that nearly $16 \%$ regressed to NGS. Similar studies were conducted in Shanghai to investigate regression to NGS with a 10 -year follow-up. They showed that $22.5 \%$ regressed from prediabetes to NGS [19]. This finding was more prominent in those following healthier lifestyles, i.e., males who exercised more and females who had smaller waist circumferences, as it is known that certain behaviors and measures will affect the outcome of prediabetes [19].

Overall, our study found borderline significance in FBS and a significant difference in HbA1c, while BMI showed no difference from the baseline. By contrast, a 12-month post-intervention lifestyle modification among Arabs resulted in significant changes in body weight, BMI, and FBS [20]. Similar to our findings, interventional studies in Saudi Arabia showed a significant reduction in HbA1c in an even shorter, sixmonth duration [21]. HbA1c at the end of follow up was $5.8 \% \pm 0.3 \%$ vs. $5.9 \%(0.5 \%)$ in the current study across glycemic groups and different interventions [21]. Moreover, a study on prediabetic overweight Japanese individuals confirmed those findings, showing the effectiveness of lifestyle modification in the prevention of DM progression [22]. Even so, other studies have established a link between lifestyle intervention and the reduction of serious complications in longer follow-ups of up to six years. This shows that lifestyle intervention in prediabetics is associated with a $47 \%$ reduction of the risk of developing retinopathy, as there was a decline in the incidence of DM in the first place [23].

It is worth noting that though initial BMI was 31.9 \pm 5.89 at the baseline, only a fraction of the cohort was referred to a dietician (15.22\%), while $4.35 \%$ were referred to bariatric surgery. Among those, no data indicated whether they actually had the surgery or visited the dietician. Thus, we cannot reflect the outcome based on referral status alone. Of those who had documented referral to a dietician in our study shows, $7.14 \%$ experienced a remission to NGS. At the same time, the majority remained in the prediabetic range. These numbers reflect those who had been referred to a dietician and do not account for lifestyle advice given during the physician interview or elsewhere, as those data might not be attained from retrospective studies. Moreover, only two-thirds of those who regressed to NGS were on metformin, suggesting that the remaining third were undocumented or had no lifestyle intervention.

Medications play an important role in regression from prediabetic to NGS. The results of a diabetes prevention program show that lifestyle modifications and metformin significantly decrease the incidence of type 2 diabetes [9]. Also, an interventional study that included 120 prediabetic participants with hypertriglyceridemia showed that $70 \%$ of participants who had metformin treatment regressed to NGS [24]. In our study, which provides an insight on the actual setting of daily practices of PHCs in SA, the most commonly prescribed medication was metformin, which might be explained by the high initial BMI of the participants, as high BMI creates more of a risk of DM progression [8]. Only $16.9 \%$ of patients with metformin showed beneficial results and regressed to NGS, whilst the majority of patients on metformin remained in the prediabetic range. Of those who progressed to DM, 58\% were on metformin. These observations might be a result of minimal medication compliance due to low perceived susceptibility or severity of the disease, as they have not developed full-blown diabetes yet. However, their adherence to metformin, perceived susceptibility, and severity were not assessed as a limitation of this study.

Gender was not a determinant factor for the outcome of this study. This supports the result of a study conducted in China, which found no association between gender and prognosis of a prediabetic state [19]. By contrast, a study conducted in Jeddah in 2016 found that DM has a strong association with a family history of diabetes, dyslipidemia in women, CVD in men, and hypertension [12]. Also, another study, conducted in Al-Kharj in SA, found that male gender, level of education, high BMI, marital status, and age above 45 had a significant predictive value with respect to prediabetes and diabetes as compared to NGS [13]. This study shows a notable relationship between obesity and prognosis. There is a higher initial BMI $35.73 \pm 5.89$ for those who progressed to diabetes compared to those who did not, although that difference was not significant, $\mathrm{p}>0.05$. Furthermore, there were no significant differences between age, dyslipidemia, hypertension, and CVD in terms of glycemic outcome. Our study cohort might not have been large enough to detect the difference, a larger sample size is needed to power the study. Predictors for progression, like advancing age, a family history of diabetes, 2-h PG, HbA1c, low HDL-cholesterol, and physical inactivity, have been observed in other studies [16]. Socioeconomic factors may impact the progression rates of 
Although prediabetes can have devastating complications, we observed a steady reduction in the number of patients following up after the first follow-up visit, which might indicate below-optimal follow-up practices. According to the World Health Organization (WHO), blood glucose screening can treat or delay diabetes and its complications; therefore, we suggest the implementation of stringent policies to improve adherence to follow-up measures or the implementation of technology-assisted DPP and weight-loss interventions to minimize the incidence of diabetes $[25,26]$.

This study faced several limitations that are worth mentioning. First, because this study used only EHRs to extract data, we found a relatively small sample size, which limits the generalization of our findings to only the capital city, Riyadh. However, we included all available prediabetic patients' data that fit the inclusion criteria. Also, this study represents the actual setting of prediabetes and the future transformation of health services in SA. A variety of possible factors-including first-degree-relative family history of DM, genetic susceptibility, physical activity, dietary intake, education, waist-to-hip ratio, and waist circumference-data were not documented as limitation for the current study and must be considered in future research $[12,19]$. Though most of the included study subjects were prescribed metformin in order to slow the progression of $\mathrm{DM}$, adherence, and compliance could not be measured and this is attributed to the nature of the retrospective study; it also limits the extrapolation of our findings to other interventions like counseling and lifestyle modification.

We encourage the physician to follow-up patients in a comprehensive approach, pay more attention to their BMI since the documented number of participants who were referred to dieticians was low, although, with a high BMI, it is better to meet with the diabetic physician and dietician simultaneously. We need more effort to make patients health-conscious, implement effective lifestyle changes to control body weight, and prevent progression to DM.

\section{Conclusions}

In this study, we found that an overwhelming majority of participants remain in the prediabetic range or progress to DM after three years of follow-ups in with different interventions. A pharmacological intervention like metformin did not significantly affect the glycemic outcome in the most of patients though adherence could not be ascertained. Prospective studies with larger sample size are needed to assess the adherence and the outcome following lifestyle modifications or pharmacological intervention, along with new modalities like technology assisted interventions.

\section{Additional Information \\ Disclosures}

Human subjects: Consent was obtained by all participants in this study. King Abdullah International Medical Research Center Institutional Review Board issued approval RC19/391/R. The study protocol was approved by King Abdullah International Medical Research Center Institutional Review Board (KAIMRC Approved). Animal subjects: All authors have confirmed that this study did not involve animal subjects or tissue. Conflicts of interest: In compliance with the ICMJE uniform disclosure form, all authors declare the following: Payment/services info: All authors have declared that no financial support was received from any organization for the submitted work. Financial relationships: All authors have declared that they have no financial relationships at present or within the previous three years with any organizations that might have an interest in the submitted work. Other relationships: All authors have declared that there are no other relationships or activities that could appear to have influenced the submitted work.

\section{References}

1. CDC: diabetes and prediabetes . (2020). Accessed: June 16, 2020: https://www.cdc.gov/chronicdisease/resources/publications/factsheets/diabetes-prediabetes.htm.

2. American Diabetes Association: Classification and diagnosis of diabetes: standards of medical care in diabetes-2019. Diabetes Care. 2019, 42:S13-S28. 10.2337/dc19-S002

3. Al-Rubeaan K, Al-Manaa HA, Khoja TA, et al.: Epidemiology of abnormal glucose metabolism in a country facing its epidemic: SAUDI-DM study. J Diabetes. 2015, 7:622-32. 10.1111/1753-0407.12224

4. Robert AA, Al Dawish MA, Braham R, Musallam MA, Al Hayek A, Al Kahtany NH: Type 2 diabetes mellitus in Saudi Arabia: major challenges and possible solutions. Curr Diabetes Rev. 2016, 13:59-64. 10.2174/1573399812666160126142605

5. Lee J, Dowd B: Effect of health information technology expenditure on patient level cost . Healthc Inform Res. 2013, 19:215-21. 10.4258/hir.2013.19.3.215

6. Huang Y, Cai X, Mai W, Li M, Hu Y: Association between prediabetes and risk of cardiovascular disease and all cause mortality: systematic review and meta-analysis. BMJ. 2016, 355:i5953. 10.1136/bmj.i5953

7. National Transformation Program. (2020). Accessed: June 16, 2020: https://vision2030.gov.sa/en/programs/NTP.

8. American Diabetes Association: Prevention or delay of type 2 diabetes: standards of medical care in diabetes -2019. Diabetes Care. 2019, 42:S29-S33. 10.2337/dc19-S003

9. Knowler WC, Barrett-Connor E, Fowler SE, Hamman RF, Lachin JM, Walker EA: Reduction in the incidence 
of type 2 diabetes with lifestyle intervention or metformin. N Engl J Med. 2002, 346:393-403. 10.1056/NEJMoa012512

10. Ramachandran A, Snehalatha C, Mary S, et al.: The Indian Diabetes Prevention Programme shows that lifestyle modification and metformin prevent type 2 diabetes in Asian Indian subjects with impaired glucose tolerance (IDPP-1). Diabetologia. 2006, 49:289-97. 10.1007/s00125-005-0097-z

11. Cho NH, Shaw JE, Karuranga S, Huang Y, Fernandes DR, Ohlrogge AW, Malanda B: IDF Diabetes Atlas: global estimates of diabetes prevalence for 2017 and projections for 2045. Diabetes Res Clin Pract. 2018, 138:271-81. 10.1016/j.diabres.2018.02.023

12. Bahijri SM, Jambi HA, Al Raddadi RM, Ferns G, Tuomilehto J: The prevalence of diabetes and prediabetes in the adult population of Jeddah, Saudi Arabia: a community-based survey. Plos One. 2016, 0152559. 10.1371/journal.pone.0152559

13. Aldossari KK, Aldiab A, Al-Zahrani JM, et al.: Prevalence of prediabetes, diabetes, and its associated risk factors among males in Saudi Arabia: a population-based survey. J Diabetes Res. 2018, 2194604. 10.1155/2018/2194604

14. Vistisen D, Kivimäki M, Perreault L, et al.: Reversion from prediabetes to normoglycaemia and risk of cardiovascular disease and mortality: the Whitehall II cohort study. Diabetologia. 2019 Aug, 62:1385-90. 10.1007/s00125-019-4895-0

15. Diabetes Prevention Program Research Group: 10-year follow-up of diabetes incidence and weight loss in the Diabetes Prevention Program Outcomes Study. Lancet. 2009, 374:1677-86. 10.1016/S01406736(09)61457-4

16. Anjana RM, Shanthi Rani CS, Deepa M, et al.: Incidence of diabetes and prediabetes and predictors of progression among Asian Indians: 10-year follow-up of the Chennai Urban Rural Epidemiology Study (CURES). Diabetes Care. 2015, 38:1441-48. 10.2337/dc14-2814

17. Regmi D, Al-Shamsi S, Govender RD, Al Kaabi J: Incidence and risk factors of type 2 diabetes mellitus in an overweight and obese population: a long-term retrospective cohort study from a Gulf state. BMJ Open. 2020, 10:e035813. 10.1136/bmjopen-2019-035813

18. Sreedharan J, Muttappallymyalil J, al Sharbatti S, et al.: Incidence of type 2 diabetes mellitus among Emirati residents in Ajman, United Arab Emirates. Korean J Fam Med. 2015, 36:253-7. 10.4082/kjfm.2015.36.5.253

19. Song X, Qiu M, Zhang X, et al.: Gender-related affecting factors of prediabetes on its 10-year outcome . BMJ Open Diabetes Res Care. 2016, 4:e000169. 10.1136/bmjdrc-2015-000169

20. Wani K, Alfawaz H, Alnaami AM, et al.: Effects of a 12-month intensive lifestyle monitoring program in predominantly overweight/obese Arab adults with prediabetes. Nutrients. 2020, 12:464. 10.3390/nu12020464

21. Al-Hamdan R, Avery A, Salter A, Al-Disi D, Al-Daghri NM, McCullough F: Identification of education models to improve health outcomes in Arab women with pre-diabetes. Nutrients. 2019, 11:1113. 10.3390/nu11051113

22. Saito T, Watanabe M, Nishida J, et al.: Lifestyle modification and prevention of type 2 diabetes in overweight Japanese with impaired fasting glucose levels: a randomized controlled trial. Arch Intern Med. 2011, 171:1352-60. 10.1001/archinternmed.2011.275

23. Gong Q, Gregg EW, Wang J, et al.: Long-term effects of a randomised trial of a 6-year lifestyle intervention in impaired glucose tolerance on diabetes-related microvascular complications: the China Da Qing Diabetes Prevention Outcome Study. Diabetologia. 2011, 54:300-7. 10.1007/s00125-010-1948-9

24. Wan Q, Wang F, Wang F, et al.: Regression to normoglycaemia by fenofibrate in pre-diabetic subjects complicated with hypertriglyceridaemia: a prospective randomized controlled trial. Diabet Med. 2010, 27:1312-17. 10.1111/j.1464-5491.2010.03107.x

25. WHO: diabetes factsheet. (2020). Accessed: July 16, 2020: https://www.who.int/news-room/factsheets/detail/diabetes.

26. Grock S, Ku J, Kim J, Moin T: A review of technology-assisted interventions for diabetes prevention . Curr Diab Rep. 2017, 17:107. 10.1007/s11892-017-0948-2 